Let $C=\bigcup_{j=0}^{\infty}\left(\left\{h\left(b_{j}\right)\right\} \cup\left\{h^{-1}\left(b_{j}\right)\right\}\right)$ and $B^{\prime}=\left\{b_{0}, b_{1}, \ldots\right\}$. Notice that $B^{\prime}$ and $C$ do not intersect. For example, if $b_{n}=h\left(b_{j}\right)$, then $n \neq j$, else $b_{n} \in N$, and $n \ngtr j$, else $b_{n} \in\left\{h\left(b_{j}\right)\right\}$, and $n \ngtr j$, else $b_{j} \in\left\{h^{-1}\left(b_{n}\right)\right\} . B^{\prime}$ is dense in $S-C$ though, for it is dense in $I$, and $M \cap(S-C)^{2}$ is closed (relative to $\left.(S-C)^{2}\right)$ and still uncountable, but it is forced to miss the $B^{\prime}$-grid in $(S-C)^{2}$.

The author is grateful to J. B. Brown and P. L. Zenor for their helpful suggestions and advice, and especially to Zenor for first raising the question of the existence of a $v^{\infty}$ space.

\title{
References
}

[1] J. B. Brown, On the Baire order of concentrated spaces and $L_{1}$ spaces, Fund. Math. 94 (1977), pp. 165-172.

[2] K. Kuratowski, Topology, Vol. I, New York-London-Warszawa 1966.

[3] E. A. Michael, Paracompactness and the Lindelöf property in finite and countable Cartesian products, Comp. Math. 23 (1971), pp. 199-214.

AUBURN UNIVERSTTY

Auburn, Alabama

Accepté par la Rédaction le 23.1. 1978

\section{Dimension of free $L$-spaces}

by

\section{Keiô Nagami (Matsuyama)}

Abstract. We introduce the class of free $L$-spaces which is countably productive and hereditary. The class is an intermediate class between that of $L$-spaces and that of $M_{1}$-spaces. The class has excellent features in dimension theory a part of which is clarified in this paper.

0. Introduction. In a previous paper [6] we introduced the notion of $L$-spaces which constitute an intermediate class between that of Lašnev spaces and that of $M_{1}$-spaces. As was noted there the class of $L$-spaces is not even finitely productive. In this paper we introduce the notion of free $L$-spaces in Section 1 which generalizes the notion of $L$-spaces. The class of free $L$-spaces is not only hereditary and countably productive but also has many excellent features in dimension theory. In Section 2 we show that even the dimension-raising theorem is valid for the class of free $L$-spaces. As trivial corollaries of this theorem there are the decomposition theorem and the coincidence theorem for two basic dimensions. A characterization theorem for a free $L$-space $X$ with $\operatorname{dim} X=n$ is also presented. Our characterization assures the existence of equi-dimensional $G_{\delta}$-envelopes as in Theorem 2.8 below. In Section 3 we show that the universal space for free $L$-spaces is the countable product of almost polyhedral spaces. As a special case we prove, in Theorem 3.8 below, that each space $X$ is a free $L$-space with $\operatorname{dim} X \leqslant 0$ if and only if it is embedded in the countable product of almost discrete spaces. Thus a role played by Baire's 0 -dimensional spaces in the theory of metric spaces is done by the countable products of almost discrete spaces in the theory of free $L$-spaces.

In this paper all spaces are assumed to be Hausdorff topological spaces, maps to be continuous onto, and images to be those under maps. The letter $N$ denotes the positive integers. For undefined terminology refer to [2] and [6].

\section{Definition of free $L$-spaces.}

1.1. Definition. Let $X$ be a space, $F$ a closed set of $X$, and $\mathscr{U}$ an anti-cover of $F$. If $S$ is a subset of $X, \mathscr{U}(S)$ denotes the star $U\{U \in \mathscr{U}: U \cap S \neq \emptyset\} . \mathscr{U}^{i}(S)$ is defined inductively by the formulae: $\mathscr{U}^{1}(S)=\mathscr{U}(S)$ and $\mathscr{U}^{i}(S)=\mathscr{U}\left(\mathscr{U}^{i-1}(S)\right)$. A set $V$ of $X$ is said to be a canonical neighborhood of $F$ (with respect to $\mathscr{U}$ ) if $V$ is an open neighborhood of $F$ such that, for each $i, \mathrm{Cl}^{i}(X-V)$ does not meet $F$. 
1.2. Definitron. For a space $X$ consider a pair $\mathscr{P}=\left(\mathscr{F},\left\{\mathscr{U}_{F}: F \in \mathscr{F}\right\}\right)$ of a $\sigma$-discrete closed collection $\mathscr{F}$ of $X$ and a collection of anti-covers $\mathscr{U}_{F}$ of $F \in \mathscr{F}$. $\mathscr{P}$ is said to be a free $L$-structure if for each point $x \in X$ and each open neighborhood $U$ of $x$ there exist a finite subcollection $\left\{F_{1}, \ldots, F_{k}\right\}$ of $\mathscr{F}$ and a canonical neighborhood $U_{i}$ of each $F_{i}$ with $x \in \bigcap_{i=1}^{k} F_{i} \subset \bigcap_{i=1}^{k} U_{i} \subset U . X$ is said to be a free $L$-space if $X$ is a paracompact space admitting a free $L$-structure.

If $\mathscr{P}$ is a free $L$-structure, the collection of all finite intersections of elements of $\mathscr{F}$ is a $\sigma$-discrete network of $X$. Thus each free $L$-space is a $\sigma$-space. Each open neighborhood of $F$ is canonical with respect to $\mathscr{U}_{F}$ if and only if $\mathscr{U}_{F}$ is approaching to $F$ in the sense of Nagami [6].

1.3. THEOREM. To be a free $L$-space is hereditary and countably productive property.

Proof. Let $X$ be a free $L$-space, $\left(\mathscr{F},\left\{\mathscr{U}_{F}: F \in \mathscr{F}\right\}\right)$ a free $L$-structure of $X$, and $S$ a subset of $X$. Since $X$ is a paracompact $\sigma$-space, $S$ is paracompact. Since the restriction $\left(\mathscr{F} \mid S,\left\{\mathscr{U}_{F} \mid S: F \in \mathscr{F}\right\}\right)$ is, as can easily be seen, a free $L$-structure of $S$, then $S$ is a free $L$-space.

Let $X_{i}, i \in N$, be a sequence of free $L$-spaces, and $\left(\mathscr{F}_{i},\left\{\mathscr{U}_{F}: F \in \mathscr{F}_{i}\right\}\right), i \in N$, a sequence of corresponding free $L$-structures. Set $X=\prod X_{i}$. Since each $X_{i}$ is a paracompact $\sigma$-space, $X$ is paracompact. Let $\pi_{i}: X \rightarrow X_{i}, i \in N$, be the projections. Set $\mathscr{H}=\bigcup \pi_{i}^{-1}\left(\mathscr{F}_{i}\right)$. Then $\mathscr{H}$ is a $\sigma$-discrete closed collection. Let us see that $\left(\mathscr{H},\left\{\pi_{i}^{-1}\left(\mathscr{U}_{F}\right): F \in \mathscr{F}_{i}, i \in N\right\}\right)$ is a free $L$-structure of $X$. If $F \in \mathscr{F}_{i}, \pi_{i}^{-1}\left(\mathscr{U}_{F}\right)$ is an anti-cover of $\pi_{i}^{-1}(F)$. Let $x=\left(x_{i}\right)$ be an arbitrary point of $X$, and $U$ an arbitrary open neighborhood of $x$. Choose a finite subset $M$ of $N$ and open neighborhoods $U_{t}$ of $x_{i}, i \in M$, such that $\bigcap\left\{\pi_{i}^{-1}\left(U_{i}\right): i \in M\right\} \subset U$. For each $i \in M$, choose a finite subcollection $\{F(i, j): j=1, \ldots, n(i)\} \subset \mathscr{F}_{i}$ and canonical neighborhoods $U(i, j)$ of $F(i, j)$ with respect to $\mathscr{U}_{F(i, j)}, j=1, \ldots, n(i)$, such that

$$
x_{i} \in \bigcap\{F(i j): j=1, \ldots, n(i)\} \subset \bigcap\{U(i, j): j=1, \ldots, n(i)\} \subset U_{i} .
$$

Then $\pi_{i}^{-1}(U(i, j))$ is a canonical neighborhood of $\pi_{i}^{-1}(F(i, j))$ with respect to $\pi_{i}^{-1}\left(\mathcal{U}_{F(i, j)}\right)$ and

$$
\begin{aligned}
x & \in \bigcap\left\{\pi_{i}^{-1}(F(i, j)): j=1, \ldots, n(i), i \in M\right\} \\
& \subset \bigcap\left\{\pi_{i}^{-1}(U(i, j)): j=1, \ldots, n(i), i \in M\right\} \\
& \subset \bigcap\left\{\pi_{i}^{-1}\left(U_{i}\right): i \in M\right\} .
\end{aligned}
$$

That completes the proof.

\section{Dimension for free $L$-spaces.}

2.1. LEMMA. Let $X$ be a hereditarily paracompact space. Let $F, H$ be a disjonit pair of closed sets of $X$. Let $\mathscr{V}$ be a $\sigma$-locally finite open cover of $X$ with $V^{5}(F) \cap H=\varnothing$. Then there exists an open set $D$ such that $F \subset D \subset \bar{D} \subset X-H$ and $\partial D \subset U\{\partial V: V \in \mathscr{V}\}$. If each binary open cover of $X$ can be refined by a $\sigma$-locally finite open cover $\mathscr{V}$ such that Ind $\partial \leqslant n-1$ for each $V \in \mathscr{V}$, then Ind $X \leqslant n$.

Cf. Nagami [2], Theorem 11.12.

2.2. Lemma (Nagami [5], Lemma 4). Let $X$ and $Y$ be paracompact $\sigma$-spaces and $f$ a closed map of $X$ onto $Y$. If order $f=n$ and, for each point $y \in Y, f^{-1}(y)$ consists of exactly $n$ points, then $\operatorname{dim} Y \leqslant \operatorname{dim} X$.

2.3. THEOREM. For a free $L$-space $X$ the following four conditions are equivalent.

(1) $\operatorname{dim} X \leqslant n$.

(2) $X$ is the image of a free $L$-space $Z$ with $\operatorname{dim} Z \leqslant 0$ under a closed map $f$ of order $\leqslant n+1$.

(3) $X$ is the sum of $n+1$ subsets $Z_{i}, i=1, \ldots, n+1$, with $\operatorname{dim} Z_{i} \leqslant 0$ for each $i$.

(4) Ind $X \leqslant n$.

Proof. The implication (2) $\rightarrow$ (3) is a direct consequence of Lemma 2.2. The implications $(3) \rightarrow(4) \rightarrow(1)$ are already known (cf. Nagami [2], Theorem 12.6).

To prove that (1) implies (2) let $\left(\mathscr{F}=\bigcup \mathscr{F}_{i},\left\{\mathscr{U}_{F}: F \in \mathscr{F}\right\}\right)$, with each $\mathscr{F}_{i}$ discrete, be a free $L$-structure of $X$. Set $\mathscr{F}_{i}=\left\{F(i, \alpha): \alpha \in A_{i}\right\}$. Let $\left\{U(i, \alpha): \alpha \in A_{i}\right\}$ and $\left\{V(i, \alpha): \alpha \in A_{i}\right\}$ be discrete collections of open sets such that

Set

$$
F(i, \alpha) \subset V(i, \alpha) \subset \mathrm{Cl} V(i, \alpha) \subset U(i, \alpha) \quad \text { for each } \alpha \in A_{i} .
$$

$$
\begin{aligned}
& H_{i}=\bigcup\left\{F(i, \alpha): \alpha \in A_{i}\right\}, \\
& U_{i}=\bigcup\left\{U(i, \alpha): \alpha \in A_{i}\right\},
\end{aligned}
$$

and

$$
V_{i}=\bigcup\left\{V(i, \alpha): \alpha \in A_{i}\right\}
$$

Then $H_{i} \subset V_{i} \subset \bar{V}_{i} \subset U_{i}$. By the perfect normality of $X$ there exists an anticover $\mathscr{V}_{i}$ of $H_{i}$ consisting of $X-\bar{V}_{i}$ and of open subsets of $U_{i}-H_{i}$ such that $V_{i}$ is a canonical neighborhood of $H_{i}$ with respect to $\mathscr{V}_{i}$. Set

$$
\mathscr{W}_{i}=\left\{X-\bar{V}_{i}\right\} \cup\left(\cup\left\{\mathscr{U}_{F(i, \alpha)}\left|(U(i, \alpha)-F(i, \alpha)) \wedge \mathscr{V}_{i}\right|(U(i, \alpha)-F(i, \alpha)): \alpha \in A_{i}\right\}\right) .
$$

Then $\mathscr{W}_{i}$ is an anti-cover of $H_{i}$. Let $\bigcup_{j=1}^{\infty} \mathscr{W}_{i j}$ be an anti-cover of $H_{i}$ refining $\mathscr{W}_{i}$ such that each $\mathscr{W}_{i j}$ is discrete in $X-H_{i}$ and $\bigcup_{j=1}^{\infty} \mathscr{W}_{i j}$ is locally finite in $X-H_{i}$. Set $\mathscr{W}_{i j}=\left\{W_{i j \beta}: \beta \in B_{i j}\right\}$ and $W_{i j}=\bigcup\left\{W_{i j \beta}: \beta \in B_{i j}\right\}$. Set $W_{i j}=\bigcup_{k=1}^{\infty} K_{i j k}$, where each $K_{i j k}$ is a closed set of $X$.

By Lèbo [1] there exist a metric space $\varrho X$ with $\operatorname{dim} \varrho X \leqslant \operatorname{dim} X$ and a contraction (i.e. a one-one map) $\varrho: X \rightarrow \varrho X$ such that the images of all $K_{i j k}$ and $X-W_{i j}$ 
under $\varrho$ are closed in $\varrho X$. Well order the collection of all disjoint pairs $\left(X-W_{i j}, K_{i j k}\right)$ as follows:

$$
\left\{\left(X-W_{i j}, K_{i j k}\right): i, j, k \in N\right\}=\left\{\left(P_{i}, Q_{i}\right): i \in N\right\} .
$$

Let $\mathscr{G}_{i}=\left\{G_{i \lambda}: \lambda \in \Lambda_{i}\right\}, i \in N$, be a sequence of locally finite open covers of $\varrho X$ satisfying the following four conditions for each $i \in N$ :

a) $\operatorname{mesh} \mathscr{G}_{i} \leqslant 1 / i$,

b) $\overline{\mathscr{G}}_{i+1}<\mathscr{G}_{i}$

c) order $\mathscr{G}_{i} \leqslant n+1$,

d) $\mathscr{G}_{i}<\left\{\varrho X-\varrho P_{i}, \varrho X-\varrho Q_{i}\right\}$.

Let $\pi_{i}^{i+1}: \Lambda_{i+1} \rightarrow \Lambda_{i}$ be a transformation such that $\pi_{i}^{i+1}(\lambda)=\mu$ yields $\bar{G}_{i+1, \lambda} \subset G_{i \mu}$, and consider the inverse system $\left\{\Lambda_{i}, \pi_{i}^{i+1}\right\}$, where each $\Lambda_{i}$ is endowed with the discrete topology. Let $\sigma Z$ be the aggregate of all points $\left(\lambda_{i}\right)$ of $\varliminf$ im $\Lambda_{i}$ with $\bigcap_{i=1}^{\infty} G_{i \lambda_{i}} \neq \varnothing$. Let $g: \sigma Z \rightarrow \varrho X$ be a transformation defined by: $g\left(\left(\lambda_{i}\right)\right)=\bigcap_{i=1}^{\infty} G_{i \lambda_{i}}$.

Then $g$ is a closed map onto with order $g \leqslant n+1$ and $\operatorname{dim} \sigma Z \leqslant 0$ by Nagami [2], Theorem 12.6. Let $\pi_{j}: \sigma Z \rightarrow \Lambda_{j}$ be the restriction of the projection of $\varliminf \mathrm{im} \Lambda_{i}$ to $\Lambda_{j}$. Consider the diagram:

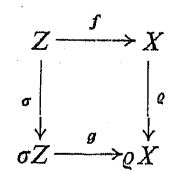

Let the set $Z$ be identical with $\varrho Z, \sigma$ the identity transformation of $Z$ onto $\sigma Z$ and $f: Z \rightarrow X$ the transformation such that $g \sigma=\varrho f$. Give $Z$ the minimal topology among those which enable both $f$ and $\sigma$ to be continuous. By an argument which is essentially the same as in Nagami [4], Theorem 6, we can see that $f$ is a closed map onto. Since $\sigma$ is a contraction, $Z$ is Hausdorff. Since order $f=\operatorname{order} g \leqslant n+1, Z$ is a paracompact space as a perfect preimage of a paracompact space $X$.

Let us construct a free $L$-structure of $Z$. Set $\mathscr{H}=\left\{\pi_{i}^{-1}(\lambda): \lambda \in \Lambda_{i}, i \in N\right\}$. Since $\mathscr{H}$ is $\sigma$-discrete and each element of $\mathscr{H}$ is open and closed, $\sigma^{-1}(\mathscr{H})$ is $\sigma$-discrete and each element of $\sigma^{-1}(\mathscr{H})$ is open and closed. Set $\mathscr{K}=\sigma^{-1}(\mathscr{H}) \wedge$ $\wedge f^{-1}(\mathscr{F})$. Then $\mathscr{K}$ is a closed collection which is $\sigma$-discrete in $Z$. Let $K$ be a generic element of $\mathscr{K}$. Then $K=\sigma^{-1} \pi_{i}^{-1}(\lambda) \cap f^{-1}(F)$ for some $i$, some $\lambda \in \Lambda_{i}$, and some
$F \in \mathscr{F}$. Set

$$
\mathscr{L}_{K}=\left\{Z-\sigma^{-1} \pi_{i}^{-1}(\lambda)\right\} \cup\left(f^{-1}\left(\mathscr{U}_{F}\right) \mid \sigma^{-1} \pi_{i}^{-1}(\lambda)\right) .
$$

Then $\mathscr{L}_{K}$ is an anti-cover of $K$.

To see that $\mathscr{P}=\left(\mathscr{K},\left\{\mathscr{L}_{\mathrm{K}}: K \in \mathscr{K}\right\}\right)$ is a free $L$-structure of $Z$ let $z$ be an arbitrary point of $Z$ and $U$ an arbitrary open neighborhood of $z$. Then

$$
z \in \sigma^{-1} \pi_{j}^{-1}(\mu) \cap f^{-1}(V) \subset U
$$

for some $j$, some $\mu \in \Lambda_{j}$, and some open neighborhood $V$ of $f(z)$. Choose a finite subcollection $\left\{F_{1}, \ldots, F_{k}\right\}$ of $\mathscr{F}$ and canonical neighborhood $D_{i}$ of $F_{i}, i=1, \ldots, k$, such that

$$
f(z) \in \bigcap_{i=1}^{k} F_{i} \subset \bigcap_{i=1}^{k} D_{i} \subset V .
$$

Set $K_{i}=\sigma^{-1} \pi_{j}^{-1}(\mu) \cap f^{-1}\left(F_{i}\right)$. Then $\sigma^{-1} \pi_{j}^{-1}(\mu) \cap f^{-1}\left(D_{i}\right)$ is a canonical neighborhood of $K_{i}$ with respect to $\mathscr{L}_{K_{i}}$. Since

$$
\begin{aligned}
& z \in \bigcap_{i=1}^{k} K_{i} \subset \bigcap_{i=1}^{k}\left(\sigma^{-1} \pi_{j}^{-1}(\mu) \cap f^{-1}\left(D_{i}\right)\right) \\
& =\sigma^{-1} \pi_{j}^{-1}(\mu) \cap f^{-1}\left(\bigcap_{i=1}^{k} D_{i}\right) \\
& \subset \sigma^{-1} \pi_{j}^{-1}(\mu) \cap f^{-1}(V) \subset U,
\end{aligned}
$$

then $\mathscr{P}$ is a free $L$-structure of $Z$.

To prove the final inequality $\operatorname{dim} Z \leqslant 0$ we need some assertions.

ASSERTION 1. $f^{-1}\left(P_{i}\right)$ and $f^{-1}\left(Q_{i}\right)$ can be separated in $Z$ by the empty set. Proof. Set $\Lambda_{j}^{\prime}=\left\{\lambda \in \Lambda_{j}: G_{j \lambda} \cap \varrho P_{j} \neq \varnothing\right\}, j \in N$. Then by the condition d), $g^{-1} \varrho P_{j} \subset \pi_{j}^{-1}\left(\Lambda_{j}^{\prime}\right) \subset \sigma Z-g^{-1} \varrho Q_{j}, \sigma^{-1} g^{-1} \varrho P_{j} \subset \sigma^{-1} \pi_{j}^{-1}\left(\Lambda_{j}^{\prime}\right) \subset Z-\sigma^{-1} g^{-1} \varrho Q_{j}$, and hence $f^{-1}\left(P_{j}\right) \subset \sigma^{-1} \pi_{j}^{-1}\left(\Lambda_{j}^{\prime}\right) \subset Z-f^{-1}\left(Q_{j}\right)$. Obviously $\sigma^{-1} \pi_{j}^{-1}\left(\Lambda_{j}^{\prime}\right)$ is open and closed. That proves the assertion.

ASSERTION 2. Let $D_{i}$ be a canonical neighborhood of $H_{i}$ with respect to $\mathscr{W}_{i}$ with $D_{i} \subset V_{i}$. Then $f^{-1}\left(H_{i}\right)$ and $Z-f^{-1}\left(D_{i}\right)$ can be separated by the empty set.

Proof. Set $E_{i}=\mathscr{W}_{i}^{5}\left(X-D_{i}\right)$ and $\mathscr{D}_{i}=\left\{f^{-1}\left(D_{i}\right)-f^{-1}\left(H_{i}\right), f^{-1}\left(E_{i}\right)\right\}$. Then $\mathscr{D}_{i}$ is a binary open cover of the subspace $Z-f^{-1}\left(H_{i}\right)$. For each $j, k \in N$, there exists, by Assertion 1, an open and closed set $R_{i j k}$ such that $f^{-1}\left(K_{i j k}\right) \subset R_{i j k} \subset f^{-1}\left(W_{i j}\right)$. Set $S_{i j k \beta}=f^{-1}\left(W_{i j \beta}\right) \cap R_{i j k}$. Then $S_{i j k \beta}$ is an open and closed set. Set

$$
\mathscr{S}_{i j k}=\left\{S_{i j k \beta}: \beta \in B_{i j}\right\}, \quad \mathscr{S}_{i}=\bigcup\left\{\mathscr{S}_{i j k}: j, k \in N\right\} .
$$

Then $\mathscr{S}_{i j k}$ is discrete, covers $R_{i j k}$, and refines $f^{-1}\left(\mathscr{W}_{i j}\right)$. Since

$$
\begin{aligned}
Z-f^{-1}\left(H_{i}\right) & =\bigcup\left\{f^{-1}\left(K_{i j k}\right): j, k \in N\right\} \\
& \subset \bigcup\left\{R_{i j k}: j, k \in N\right\} \\
& \subset \bigcup\left\{f^{-1}\left(W_{i j}\right): j \in N\right\}=Z-f^{-1}\left(H_{i}\right),
\end{aligned}
$$

$\mathscr{S}_{i}$ is a $\sigma$-discrete cover of $Z-f^{-1}\left(H_{i}\right)$ whose elements are open and closed. Since $\mathscr{S}_{i}<\bigcup_{j=1}^{\infty} f^{-1}\left(\mathscr{W}_{i j}\right)<f^{-1}\left(\mathscr{W}_{i}\right)$, there exists, by Lemma 2.1, a set $D$, being open and $j=1$
closed in $Z-f^{-1}\left(H_{i}\right)$, such that

$$
Z-f^{-1}\left(E_{i} \cup H_{i}\right) \subset D \subset f^{-1}\left(D_{i}\right)-f^{-1}\left(H_{i}\right) \ldots .
$$


Since $Z-f^{-1}\left(E_{i}\right)$ is a canonical neighborhood of $f^{-1}\left(H_{i}\right)$ with respect to $f^{-1}\left(\mathscr{W}_{i}\right)$, $D \cup f^{-1}\left(H_{i}\right)$, is a canonical neighborhood of $f^{-1}\left(H_{i}\right)$ with respect to $f^{-1}\left(\mathscr{W}_{i}\right)$. The latter fact implies that $D \cup f^{-1}\left(H_{i}\right)$ is open and closed in $Z$. The inequalities $f^{-1}\left(H_{i}\right) \subset Z-f^{-1}\left(E_{i}\right) \subset D \cup f^{-1}\left(H_{i}\right) \subset f^{-1}\left(D_{i}\right)$ proves the assertion.

ASSERTION 3. For each point $x \in X$ and each open neighborhood $U$ of $x$ there exist finite elements $F\left(i, \alpha_{i}\right) \in \mathscr{F}_{i}, i \in M$, and an open and closed set $V$ of $Z$ such that

$$
f^{-1}(x) \subset \bigcap_{i \in M} f^{-1}\left(F\left(i, \alpha_{i}\right)\right) \subset V \subset\left(\bigcap_{i \in M} f^{-1}\left(V\left(i, \alpha_{i}\right)\right)\right) \cap f^{-1}(U) .
$$

Proof. Choose a finite set $M \subset N$, elements $F\left(i, \alpha_{i}\right) \in \mathscr{F}_{i}, i \in M$, and canonical neighborhoods $G_{i}$ of $F\left(i, \alpha_{i}\right)$ with respect to $\mathscr{U}_{F\left(i, \alpha_{i}\right)}, i \in M$, such that

Notice that

$$
x \in \bigcap_{i \in M} F\left(i, \alpha_{i}\right) \subset \bigcap_{i \in M} G_{i} \subset U .
$$

$$
\mathscr{W}_{i}\left|\left(U\left(i, \alpha_{i}\right)-F\left(i, \alpha_{i}\right)\right)<\mathscr{U}_{F\left(i, \alpha_{i}\right)}\right|\left(U\left(i, \alpha_{i}\right)-F\left(i, \alpha_{i}\right)\right)
$$

and $V\left(i, \alpha_{i}\right)$ is a canonical neighborhood of $F\left(i, \alpha_{i}\right)$ with respect to

$$
\mathscr{W}_{i} \mid\left(U\left(i, \alpha_{i}\right)-F\left(i, \alpha_{i}\right)\right) \text {, }
$$

then $G_{i} \cap V\left(i, \alpha_{i}\right)$ is a canonical neighborhood of $F\left(i, \alpha_{i}\right)$ with respect to $\mathscr{W}_{i} \mid\left(U\left(i, \alpha_{i}\right)-F\left(i, \alpha_{i}\right)\right)$. Therefore, if we set

$$
S_{i}=\left(G_{i} \cap V\left(i, \alpha_{i}\right)\right) \cup\left(\bigcup\left\{V(i, \alpha): \alpha \neq \alpha_{i}\right\}\right),
$$

$S_{i}$ is a canonical neighborhood of $H_{i}$ with respect to $\mathscr{W}_{i}$. By Assertion 2 there exists an open and closed set $T_{i}$ of $Z$ with $f^{-1}\left(H_{i}\right) \subset T_{i} \subset f^{-1}\left(S_{i}\right)$. If we set $T_{i}^{\prime}=T_{i} \cap f^{-1}\left(V\left(i, \alpha_{i}\right)\right), T_{i}^{\prime}$ is still an open and closed set of $Z$ with $f^{-1}\left(F\left(i, \alpha_{i}\right)\right)$ $\subset T_{i}^{\prime} \subset f^{-1}\left(G_{i} \cap V\left(i, \alpha_{i}\right)\right)$. Set $V=\bigcap_{i \in M} T_{i}^{\prime}$. Then

$$
\begin{aligned}
f^{-1}(x) & =\bigcap_{i \in M} f^{-1}\left(F\left(i, \alpha_{i}\right)\right) \subset V \\
& \subset \bigcap_{i \in M} f^{-1}\left(G_{i} \cap V\left(i, \alpha_{i}\right)\right) \\
& d\left(\bigcap_{i \in M} f^{-1}\left(V\left(i, \alpha_{i}\right)\right)\right) \cap f^{-1}(U) .
\end{aligned}
$$

That proves the assertion.

Assertion 4. Let $U$ be an open set of $X$. Then $f^{-1}(U)$ admits a cover $\mathscr{W}$ which is $\sigma$-discrete in $Z$ and each element of which is open and closed.

Proof. Set

$$
\begin{aligned}
N^{*} & =\{M \subset N:|M|<\infty\} \\
A_{M} & =\prod\left\{A_{i}: i \in M\right\}, M \in N^{*} \\
F_{\gamma} & =\bigcap\left\{F\left(i, \alpha_{i}\right): i \in M\right\}, \gamma=\left(\alpha_{i}: i \in M\right) \in A_{M}, M \in N^{*} \\
V_{\gamma} & =\bigcap\left\{V\left(i, \alpha_{i}\right): i \in M\right\}, \gamma=\left(\alpha_{i}: i \in M\right) \in A_{M}, M \in N^{*}
\end{aligned}
$$
Let $B_{M}$ be the aggregate of all indices $\gamma \in A_{M}$ such that $f^{-1}\left(F_{\gamma}\right) \subset W_{\gamma} \subset f^{-1}\left(V_{\gamma} \cap U\right)$
for some open and closed set $W_{\gamma}$. Set

$$
\mathscr{W}=\left\{W_{\gamma}: \gamma \in B_{M}, M \in N^{*}\right\} .
$$

By Assertion 3, $\left\{f^{-1}\left(F_{\gamma}\right): \gamma \in B_{M}, M \in N^{*}\right\}$ covers $f^{-1}(U)$ and hence $\mathscr{W}$, consisting of open and closed sets, covers $f^{-1}(U)$. Since $\left\{V_{\gamma}: \gamma \in A_{M}\right\}$ is discrete in $X$ for each $M \in N^{*}$ and $N^{*}$ is countable, $\left\{f^{-1}\left(V_{\gamma}\right): \gamma \in B_{M}, M \in N^{*}\right\}$ is $\sigma$-discrete in $Z$ and hence $\mathscr{W}$ is $\sigma$-discrete in $Z$. That proves the assertion.

ASSERTION 5. Let $G$ be an open set of $Z$. Then $G$ has a cover $\mathscr{G}$ which is $\sigma$-discrete in $Z$ and each element of which is open and closed.

Proof. For each $i$ and each $\lambda \in \Lambda_{i}$ let $U_{\lambda}$ be the largest open set of $X$ such that $\sigma^{-1} \pi_{i}^{-1}(\lambda) \cap f^{-1}\left(U_{\lambda}\right) \subset G$. Then $G=\bigcup\left\{\sigma^{-1} \pi_{i}^{-1}(\lambda) \cap f^{-1}\left(U_{\lambda}\right): \lambda \in \Lambda_{i}, i \in N\right\}$. By Assertion 4 there exists a cover $\mathscr{W}$, of $f^{-1}\left(U_{\lambda}\right)$ which is $\sigma$-discrete in $Z$ and each element of which is open and closed. Set

$$
\mathscr{G}_{\lambda}=\mathscr{H}_{\lambda} \mid \sigma^{-1} \pi_{i}^{-1}(\lambda) \cap f^{-1}\left(U_{\lambda}\right) .
$$

Then $\mathscr{G}_{\lambda}$ is still $\sigma$-discrete in $Z$ and each element of $\mathscr{G}_{\lambda}$ is open and closed. Set

$$
\mathscr{G}=\bigcup\left\{\mathscr{G}_{\lambda}: \lambda \in \Lambda_{i}, i \in N\right\} \text {. }
$$

Then $\mathscr{G}$ is a cover of $G$ consisting of open and closed sets. Since $\sigma^{-1}(\mathscr{H})$ is $\sigma$-discrete, $\mathscr{G}$ is also $\sigma$-discrete. That proves the assertion.

By Assertion 5 each binary open cover of $Z$ can be refined by a cover which is $\sigma$-discrete in $Z$ and each element of which is open and closed. That implies $\operatorname{dim} Z \leqslant 0$ by Lemma 2.1. The proof of the theorem is thus completed.

From the above argument and from the first half of Theorem 1.3 it can easily be seen that the restriction of $\left\{\left(K_{i j k}, X-W_{i j}\right): i, j, k \in N\right\}$ to an arbitrary subset $S$ of $X$ determines Ind $S$. Thus we have the following which is a generalization of Nagami [6], Lemma 3.5.

2.4. THEOREM. A free $L$-space $X$ admits a countable collection of disjoint pairs of closed sets determining Ind of all subsets of $X$.

2.5. Definition. Let $X$ be a space. Let $\mathscr{F}_{i}=\left\{F(i, \alpha): \alpha \in A_{i}\right\}$ be a discrete collection of closed sets of $X$. Let $\mathscr{V}_{i}=\left\{V(i, \alpha): \alpha \in A_{i}\right\}$ be a discrete collection of open sets with $F(i, \alpha) \subset V(i, \alpha), \alpha \in A_{i}$. Let $\mathscr{U}_{i}$ be an anti-cover of

$$
H_{i}=\bigcup\left\{F(i, \alpha): \alpha \in A_{i}\right\} \text {. }
$$

Then $\left(\mathscr{F}=\cup \mathscr{F}_{i}, \cup \mathscr{V}_{i}, \cup \mathscr{U}_{i}\right)$ is said to be a free $L$-mesh of $X$ if the following two conditions are satisfied:

a) $\bigcup\left\{V(i, \alpha): \alpha \in A_{i}\right\}$ is a canonical neighborhood of $H_{i}$ with respect to $\mathscr{U}_{i}$.

b) For each point $x \in X$ and each open neighborhood $U$ of $x$ there exist finite elements $F\left(i, \alpha_{i}\right) \in \mathscr{F}, i \in M$, and canonical neighborhoods $V_{i}$ of $H_{i}$ with respect 
to $\mathscr{U}_{i}, i \in M$, such that

$$
x \in \bigcap_{i \in M} F\left(i, \alpha_{i}\right) \subset \bigcap_{i \in M}\left(V_{i} \cap V\left(i, \alpha_{i}\right)\right) \subset U .
$$

A free $L$-mesh is said to be a strict free $L$-mesh if it satisfies one more condition: is $H_{i}$.

c) The intersection of all canonical neighborhoods of $H_{i}$ with respect to $\mathscr{U}_{i}$

2.6. LEMMA. A paracompact space $X$ is a free $L$-space if and only if $X$ admits a (strict) free $L$-mesh.

The necessity is essentially proved in Theorem 2.3. The sufficiency is an easy exercise.

2.7. Theorem. A paracompact space $X$ is a free $L$-space with $\operatorname{dim} X \leqslant n$ if and only if $X$ admits a (strict) free $L-$ mesh $\left(\cup \mathscr{F}_{i}, \cup \mathscr{V}_{i}, \cup \mathscr{U}_{i}\right)$ such that each $\mathscr{U}_{i}$ is locally finite in $X-H_{i}$ and $\operatorname{dim} \partial U \leqslant n-1$ for each element $U$ of $\cup \mathscr{U}_{i}$.

This is also an easy exercise by virtue of Lemmas 2.1 and 2.6, if we apply an analogous argument to that in Assertion 4 of Theorem 2.3 .

2.8. Theorem. Let $X$ be a free $L$-space and $Y$ a subset of $X$ with $\operatorname{dim} Y \leqslant n$. Then there exists a $G_{\delta}$-set $S$ with $Y \subset S$ and $\operatorname{dim} S \leqslant n$.

Proof. By Theorem 2:3 there exist subsets $Z_{i}, i=1, \ldots, n+1$, of $Y$ with

$Y=\bigcup_{i=1}^{n+1} Z_{i}$ and with $\operatorname{dim} Z_{i} \leqslant 0$. Let $\left(\bigcup \mathscr{F}_{i}, \cup \mathscr{V}_{i}, \cup \mathscr{U}_{i}\right)$ be a strict free $L$-mesh of $X$, where each $\mathscr{U}_{i}$ is locally finite in $X-H_{i}$. Set $\mathscr{U}_{i}=\left\{U(i, \beta): \beta \in B_{i}\right\}$. Let $\mathscr{P}_{i}=\left\{P(i, \beta): \beta \in B_{i}\right\}$ be a closed cover of $X-H_{l}$ with $P(i, \beta) \subset U(i, \beta)$ for each $\beta \in B_{i}$. Since the mesh is strict, $\mathrm{Cl} U(i, \beta) \cap H_{i}=\varnothing$ and hence $P(i, \beta)$ is closed in $X$. Since $\operatorname{dim} Z_{1} \leqslant 0$, there exists an open set $Q(i, \beta)$ such that $P(i, \beta) \subset Q(i, \beta)$ $\subset \mathrm{Cl} Q(i, \beta) \subset U(i, \beta)$ and $\partial Q(i, \beta) \cap Z_{1}=\varnothing$. Set

$$
R_{i}=\bigcup\left\{\partial Q(i, \beta): \beta \in B_{i}\right\} .
$$

Then $R_{i}$ is closed in $X-H_{i}$. Since $H_{i}$ is $G_{\delta}, R_{i}$ is an $F_{\delta}$-set of $X$. Set $S_{1}=X-\bigcup_{i=1}^{\infty} R_{i}$. Then $S_{1}$ is a $G_{\delta}$-set of $X$ with $Z_{1} \subset S_{1}$. Set $\mathscr{Q}_{i}=\left\{Q(i, \beta): \beta \in B_{i}\right\}$. Let $\mathscr{T}=\left(\bigcup \mathscr{F}_{i}^{\prime}, \cup \mathscr{V}_{i}^{\prime}, \cup \mathscr{Q}_{i}^{\prime}\right)$ be the restriction of $\left(\bigcup \mathscr{F}_{i}, \cup \mathscr{V}_{i}, \bigcup \mathscr{Q}_{i}\right)$ to $S_{1}$. Then $\mathscr{T}$ is a strict free $L$-mesh of $S_{1}$ such that $\partial Q^{\prime}=\varnothing$ for each element $Q^{\prime}$ of $\cup \mathscr{Q}_{i}^{\prime}$. Thus $\operatorname{dim} S_{1} \leqslant 0$ by Theorem 2.6 .

Let $S_{i}, i=2, \ldots, n+1$, be $G_{\delta}$ sets of $X$ with $\operatorname{dim} S_{i} \leqslant 0$ and $Z_{i} \subset S_{i}$. Set $S=\bigcup^{n+1} S_{i}$. Then $S$ is $G_{\delta}$ set of $X$ with $\operatorname{dim} S \leqslant n$ and $Y \subset S$. That completes the proof.

2.9. THEOREM. For a separable free $L$-space $X$ the following four conditions are equivalent.

(1) $\operatorname{dim} X \leqslant n$.

(2) $X$ is the image of a separable free $L$-space $Z$ with $\operatorname{dim} Z \leqslant 0$ under a closed map of order $\leqslant n+1$.
(3) Ind $X \leqslant n$

(4) ind $X \leqslant n$.

Proof. For a separable $X, \varrho X$ in the diagram in Theorem 2.2 has to be separable. Then we can assume that $\sigma Z$ is separable. In that case the pullback $Z$ in the diagram has to be separable too. Thus the implications $(1) \rightarrow(2) \rightarrow(3) \rightarrow(1)$ are true. When $X$ is separable, $X$ is Lindelöf. Hence for such $X$, as is well known, ind $X=$ Ind $X$. That completes the proof.

2.10. Theorem. Let $X$ be a (separable) free $L$-space. Then $X$ is the perfect image of a (separable) free $L$-space $Z$ with $\operatorname{dim} Z \leqslant 0$.

This is essentially proved in Theorem 2.3 , where we have to assume that $g$ in the diagram is merely perfect. In that case $f$ in the diagram has to be perfect.

2.11. Problem. Is the perfect image of a free $L$-space again a free $L$-space?

2.12. Problem. Let $X$ be a Lašnev space. Is $X$ the perfect image of a Lašnev space $Z$ with $\operatorname{dim} Z \leqslant 0$ ? If moreover $\operatorname{dim} X \leqslant n$, is $X$ the image of a Lašnev space $Z$ with $\operatorname{dim} Z \leqslant 0$ under a closed map $f$ of order $\leqslant n+1$ ?

3. Embedding theorems for free $L$-spaces.

3.1. Definition. Let $X$ be a space. The set of all points of $X$ which have metric neighborhoods is said to be the metric part of $X$. The complement of the metric part is said to be the nonmetric part. $X$ is said to be an almost metric space if the following three conditions are satisfied:

a) $X$ is. perfectly normal and paracompact.

b) The collection of points of the nonmetric part $X_{0}$ is discrete.

c) $X_{0}$ has an anti-cover approaching to $X_{0}$.

An almost metric space is said to be an almost discrete space if its metric part is discrete as a relative space.

3.2. LemMa. An almost metric space $X$ is an $L$-space.

Proof. $X$ is of course a $\sigma$-space. Let $X_{0}$ be the nonmetric part of $X$. Let $\mathscr{U}$ be an anti-cover of $X_{0}$ approaching to $X_{0}$. Let $F$ be an arbitrary closed set of $X$. Let $U$ be an open set of $X$ with $X_{0}-F \subset U \subset \bar{U} \subset X-F$. Let $\mathscr{V}$ be an open cover of $X-X_{0} \cup F$ approaching to $F-X_{0}$ in $X-X_{0}$. Set $\mathscr{W}=\mathscr{U} \wedge \mathscr{V}$. Then $\mathscr{W}$ is an open cover of $X-X_{0} \cup F$. Set $\mathscr{G}=\mathscr{W} \cup\{U\}$. Then $\mathscr{G}$ is an anti-cover of $X-F$

To prove that $\mathscr{G}$ is approaching to $F$ let $G$ be an arbitrary open neighborhood of $F$. Since $\mathscr{T}$ is approaching to $X_{0}$, there exists an open neighborhood $D$ of $F \cap X_{0}$ such that $D \cap \mathscr{U}(X-G)=\varnothing$ and hence $D \cap \mathscr{W}(X-G)=\varnothing$. Since $\mathscr{V}$ is approaching to $F-X_{0}$ in $X-X_{0}$, there exists an open set $E$ of $X-X_{0}$ with $F-X_{0} \subset E$ and $E \cap \mathscr{V}(X-G)=\varnothing$. Then $E \cap \mathscr{W}(X-G)=\varnothing$. Set $T=D \cup E-\bar{U}$. Then $T$ is an open neighborhood of $F$ with $T \cap \mathscr{G}(X-G)=\varnothing$, which implies that $\mathscr{G}$ is approaching to $F$. That completes the proof.

3.3. Definition. In this paper polyhedra are simplicial polyhedra with the metric topology. Let $K$ be a polyhedron and $A$ the vertex-set of $K$. Let the star of 
$\alpha \in A$, say $\operatorname{St}(\alpha)$, be the set of all points of $K$ whose barycentric weights on $\alpha$ are positive. The star-cover of $K$ is $\{\operatorname{St}(\alpha): \alpha \in A\}$. An almost metric space $X$ is said to be an almost polyhedral space if the following two conditions are satisfied:

a) The metric part of $X$ is a polyhedron $K$.

b) The star-cover of $K$ is approaching to the nonmetric part of $X$.

3.4. THEOREM. For a space $X$ the following four statements are equivalent.

(1) $X$ is a free $L$-space.

(2) $X$ is embedded in the countable product of almost polyhedral spaces.

(3) $X$ is embedded in the countable product of almost metric spaces.

(4) $X$ is embedded in the countable product of $L$-spaces.

Proof. That (2) implies (3) is clear. The implications (3) $\rightarrow$ (4) and (4) $\rightarrow$ (1) are assured to be true respectively by Lemma 3.2 and by Theorem 1.3.

To prove that (1) implies (2) let $\left(\bigcup \mathscr{F}_{i}, \cup \mathscr{V}_{i}, \cup \mathscr{U}_{i}\right)$ be a strict free $L$-mesh of $X$, where
a) $\mathscr{F}_{i}=\left\{F(i, \alpha): \alpha \in A_{i}\right\}$,
b) $\mathscr{V}_{i}=\left\{V(i, \alpha): \alpha \in A_{i}\right\}$,
c) $\mathscr{U}_{i}=\left\{U(i, \beta): \beta \in B_{i}\right\}$,
d) $H_{i}=\bigcup\left\{F(i, \alpha): \alpha \in A_{i}\right\}$,
e) $\mathscr{U}_{i}$ is locally finite in $X-H_{i}$,
f) $H_{i}$ is the countable intersection of its canonical neighborhoods with respect to $\mathscr{U}_{i}$,

g) $\left\{\mathscr{U}_{i}(V(i, \alpha)): \alpha \in A_{i}\right\}$ is discrete.

Let $K_{i}$ be the nerve of $\mathscr{U}_{i}$ and $f_{i}: X-H_{i} \rightarrow K_{i}$ be a Kuratowski map defined as follows: The vertex-set of $K_{i}$ is $B_{i} . \varphi_{\beta}: X-H_{i} \rightarrow I$ is a map such that $\varphi_{\beta}(x)>0$ if and only if $x \in U(i, \beta)$ and such that $\left\{\varphi_{\beta}: \beta \in B_{i}\right\}$ is a partition of unity. $f_{i}(x)$ is the point of $K_{i}$ whose barycentric weight on $\beta \in B_{i}$ is $\varphi_{\beta}(x)$, i. e. $f_{i}(x)=\sum\left\{\varphi_{\beta}(x) \beta: \beta \in B_{i}\right\}$. Introduce $K_{i}$ the metric topology $\mathscr{D}_{i}$. Then as is well known $f_{i}$ is continuous.

Let $X_{i}$ be the disjoint sum of $K_{i}$ and $A_{i}$. Set $\mathscr{S}_{i}=\left\{\operatorname{St}(\beta): \beta \in B_{i}\right\}$. Let $g_{i}: X \rightarrow X_{i}$ be a transformation defined by:

$$
g_{i} \mid X-H_{i}=f_{i}, \quad g_{i}(x)=\alpha, \quad x \in F(i, \alpha) .
$$

To give $X_{i}$ a suitable topology we need some notations. Set

$$
\begin{aligned}
& B(E)=\left\{\beta \in B_{i}: U(i, \beta) \subset E\right\}, \quad E \subset X, \\
& {[E]=(\bigcup\{U(i, \beta): \beta \in B(E)\}) \cup\left(E \cap H_{i}\right) .}
\end{aligned}
$$

Let $\mathscr{E}_{i}=\left\{E_{\lambda}: \lambda \in \Lambda_{i}\right\}$ be the collection of all canonical neighborhoods of $H_{i}$ with $E_{\lambda}=\left[E_{\lambda}\right]$. Set

$$
\begin{aligned}
& G_{\lambda}=A_{i} \cup\left(\cup\left\{\operatorname{St}(\beta): \beta \in B\left(E_{\lambda}\right)\right\}\right), \\
& \mathscr{G}_{i}=\left\{G_{\lambda}: \lambda \in \Lambda_{i}\right\},
\end{aligned}
$$

$$
\begin{aligned}
W_{\alpha} & =\{\alpha\} \cup(\cup\{\operatorname{St}(\beta): \beta \in B(V(i, \alpha))\}), \quad \alpha \in A_{i}, \\
\mathscr{W}_{i} & =\left\{W_{\alpha}: \alpha \in A_{i}\right\}, \\
\mathscr{B}_{i} & =\mathscr{D}_{i} \cup\left(\mathscr{G}_{i} \wedge \mathscr{W}_{i}\right) .
\end{aligned}
$$

Give $X_{i}$ the topology having $\mathscr{B}_{i}$ as a base. Since $g_{i}^{-1}\left(G_{\lambda}\right)=E_{\lambda}, \lambda \in \Lambda_{i}$, and $g_{i}^{-1}\left(W_{\alpha}\right)=[V(i, \alpha)], \alpha \in A_{i}$, then $g_{i}$ is continuous.

ASSERTION 1. If $E$ is a canonical neighborhood of $H_{i}$, then $[E]$ is also canonical. Proof. The assertion follows at once from the inequalities $X-\mathscr{U}_{i}(X-E)$ $\subset[E] \subset E$.

ASSERTION 2. $\mathscr{W}_{i}$ is discrete.

Proof. Assume that there exist distinct elements $\alpha, \alpha^{\prime} \in A_{i}$ and $\operatorname{St}(\beta)$ such that $\operatorname{St}(\beta) \cap W_{x} \cap W_{\alpha^{\prime}} \neq \varnothing$. Then there exist $\beta_{1} \in B(V(i, \alpha))$ and $\beta_{2} \in B\left(V\left(i, \alpha^{\prime}\right)\right)$ such that $\operatorname{St}(\beta) \cap \operatorname{St}\left(\beta_{1}\right) \neq \varnothing$ and $\operatorname{St}(\beta) \cap \operatorname{St}\left(\beta_{2}\right) \neq \varnothing$. Thus $\left\{\beta, \beta_{1}\right\}$ and $\left\{\beta, \beta_{2}\right\}$ span 1-simplices of $K_{i}$ and hence $U(i, \beta) \cap U\left(i, \beta_{1}\right) \neq \varnothing$ and $U(i, \beta) \cap U\left(i, \beta_{2}\right) \neq \varnothing$. These two inequalities imply that $\mathscr{U}_{i}(V(i, \alpha)) \cap V\left(i, \alpha^{\prime}\right) \neq \varnothing$, which contradicts to $\mathrm{g})$.

ASSERTION 3. For each element $E_{\mu} \in \mathscr{E}_{i}$, there exists an element $E_{\lambda} \in \mathscr{E}_{i}$ with $\mathscr{U}_{i}\left(E_{\lambda}\right) \cup H_{i} \subset E_{\mu}$.

Proof. Set $E_{\lambda}=\left[X-\mathrm{Cl}_{i}\left(X-E_{\mu}\right)\right]$. Then $E_{\lambda}$ is the required.

AsSERTION 4. The inequality $\mathscr{U}_{i}\left(E_{\lambda}\right) \cup H_{i} \subset E_{\mu}, \lambda, \mu \in \Lambda_{i}$, implies that $\mathscr{S}_{i}\left(G_{\lambda}\right) \cup$ $\cup A_{i} \subset G_{\mu}$ and hence $\bar{G}_{\lambda} \subset G_{\mu}$.

Proof. Let $\beta \in B_{i}$ be an index with $\operatorname{St}(\beta) \cap G_{\lambda} \neq \varnothing$. Then there exists $\beta_{1} \in B\left(E_{\lambda}\right)$ with $\operatorname{St}(\beta) \cap \operatorname{St}\left(\beta_{1}\right) \neq \varnothing$. Hence $U(i, \beta) \cap U\left(i, \beta_{1}\right) \neq \varnothing$, which implies that $U(i, \beta) \subset \mathscr{U}_{i}\left(E_{\lambda}\right)$ and hence $\beta \in B\left(E_{\mu}\right)$. Thus $\operatorname{St}(\beta) \subset G_{\mu}$.

ASSERTION 5. $X_{i}$ is Hausdorff.

The assertion can be seen by f), Assertions 1, 2, 3, 4 and by the fact that the topology of $X_{i}$ does not disturb the topology of $K_{i}$.

Assertion 6. $A_{i}$ is a $G_{z^{-}}$set of $X_{i}$.

Proof. By f) there exists a sequence $E_{\lambda_{j}}, j \in N$, of elements of $\mathscr{E}_{i}$ such that $H_{i}=\bigcap_{j=1}^{\infty} E_{\lambda_{j}}$. By Assertion 3 we can pick $E_{\mu_{j}} \in \mathscr{E}_{i}$ with $\mathscr{U}_{i}\left(E_{\mu_{j}}\right) \subset E_{\lambda_{j}}$. Let $\operatorname{St}(\beta)$ be an arbitrary element of $\mathscr{S}_{i}$. Then there exists $k$ with $U(i, \beta)-E_{\lambda_{k}} \neq \varnothing$ and hence with $U(i, \beta) \cap E_{\mu_{k}}=\varnothing$. Thus for each $\beta^{\prime} \in B\left(E_{\mu_{k}}\right), U(i, \beta) \cap U\left(i, \beta^{\prime}\right)=\varnothing$. Therefore $\operatorname{St}(\beta) \cap \operatorname{St}\left(\beta^{\prime}\right)=\varnothing$ for each $\beta^{\prime} \in B\left(E_{\mu_{k}}\right)$ and hence $\operatorname{St}(\beta) \cap G_{\mu_{k}}=\varnothing$. That proves the equality $A_{i}=\bigcap_{j=1}^{\infty} G_{\mu_{j}}$.

ASSERTION 7. $X_{i}$ is paracompact and perfectly normal.

$\operatorname{Proof}$. Let $\mathscr{U}$ be an arbitrary open cover of $X_{i}$. For each $\alpha \in A_{i}$ choose $U_{\alpha} \in \mathscr{U}$ with $\alpha \in U_{\alpha}$, and then choose $\lambda(\alpha) \in \Lambda_{i}$ with $W_{\alpha} \cap G_{\lambda(\alpha)} \subset U_{\alpha}$. Set

$$
E=\bigcup\left\{g_{i}^{-1}\left(W_{\alpha} \cap G_{\lambda(\alpha)}\right): \alpha \in A_{i}\right\} \text {. }
$$


Then $E$ is a canonical neighborhood of $H_{i}$. By Assertions 2 and 3, there exist elements $\mu, v \in \Lambda_{i}$ with $E_{\mu} \subset E$ and $\bar{G}_{v} \subset G_{\mu}$. Let $\mathscr{V}$ be a locally finite open cover of $X_{i}-G_{v}$ refining $\mathscr{U}$. Set

$$
\mathscr{W}=\left\{W_{\alpha} \cap G_{\lambda(\alpha)}: \alpha \in A_{i}\right\}, \quad \mathscr{D}=\mathscr{W} \cup \mathscr{V} \mid\left(X-\bar{G}_{v}\right) .
$$

Then by Assertion 2, $\mathscr{D}$ is a locally finite open cover of $X_{i}$ refining $\mathscr{U}$. Thus $X_{i}$ is a paracompact space by Assertion 5 and hence normal.

Let $U$ be an arbitrary open set of $X_{i}$. By Assertion 4 there exists a sequence $G_{j}, j \in N$, of open sets of $X_{i}$ with $A_{i}=\bigcap_{j=1}^{\infty} G_{j}$. Let $F_{j k}, k \in N$, be a sequence of closed sets of $X_{i}$ such that $U-G_{j}=\bigcup_{k=1}^{\infty} F_{j k}$. Then

$$
U=\left(\bigcup_{j, k=1}^{\infty} F_{j k}\right) \cup\left(U \cap A_{i}\right) .
$$

Since $U \cap A_{i}$ is closed, $U$ is an $F_{\sigma}$-set of $X_{i}$.

ASSERTION 8. $\mathscr{S}_{i}$ is approaching to $A_{i}$.

This is clear from Assertions 3 and 4.

ASSERTION 9. Let $g: X \rightarrow \prod_{i=1}^{\infty} X_{i}$ be a transformation defined by: $g(x)=\left(g_{i}(x)\right)$. Then $g$ is an embedding.

Proof. Since each $g_{i}$ is continuous, $g$ is continuous. To prove $g$ is one-one let $x, y$ be distinct points of $X$. Then for some $j \in N$, some canonical neighborhood $V_{j}$ of $H_{j}$ with respect to $\mathscr{U}_{j}$, and some $\alpha \in A_{j}$,

$$
x \in F(j, \alpha) \subset V_{j} \cap(V(j, \alpha)) \subset X-\{y\} .
$$

When $y \notin V(j, \alpha), g(y) \notin W_{\alpha}$ and hence $g_{j}(y) \neq \alpha$. Since $g_{j}(x)=\alpha, g_{j}(x) \neq g_{j}(y)$ and hence $g(x) \neq g(y)$.

Consider the case when $y \notin V_{j}$. Choose $\lambda \in \Lambda_{j}$ such that $E_{\lambda} \subset V_{j}$. Since

$g_{j}^{-1}\left(G_{\lambda}\right)=E_{\lambda}, g_{j}(y) \notin G_{\lambda}$. Since $g_{j}(x)=\alpha \in G_{\lambda}, g_{j}(x) \neq g_{j}(y)$ and hence $g(x) \neq g(y)$.
To prove the continuity of $g^{-1}$ let $x$ be an arbitrary point of $X$ and $U$ an arbitrary open neighborhood of $x$. Choose finite elements $F\left(i, \alpha_{i}\right) \in \bigcup \mathscr{F}_{j}, j \in M$, and elements $\lambda_{i} \in A_{i}, i \in M$, such that

Set

$$
x \in \bigcap_{i \in M} F\left(i, \alpha_{i}\right) \subset \bigcap_{i \in M}\left(E_{\lambda_{i}} \cap\left[V\left(i, \alpha_{i}\right)\right]\right) \subset U
$$

$$
W=\prod_{i \in M}\left(G_{\lambda_{i}} \cap W_{\alpha_{i}}\right) \times \prod_{i \in N-M} X_{i}
$$

Since

$$
g_{i}^{-1}\left(G_{\lambda_{i}} \cap W_{\alpha_{i}}\right)=E_{\lambda_{1}} \cap\left[V\left(i, \alpha_{i}\right)\right], \quad i \in M, \quad g^{-1}(W) \subset U .
$$

Thus $g^{-1}$ is continuous.
ASSERTION 10. $X_{i}$ is embedded in the countable product of almost polyhedral spaces.

Proof. If $X_{i}$ is not first-countable at any $\alpha \in A_{i}$, then $X_{i}$ itself is almost polyhedral and there is no problem. Let $A_{i}^{\prime}$ be the set of all points $\alpha \in A_{i}$ at which $X_{i}$ is not first-countable. Set $A_{i}^{\prime \prime}=A_{i}-A_{i}^{\prime}$. Then it can easily be seen that $K_{i} \cup A_{i}^{\prime \prime}$ is metric and $A_{i}^{\prime}$ is the nonmetric part of $X_{i}$. Let $\left\{P(j, \alpha): \alpha \in A_{i}^{\prime \prime}, j \in N\right\}$ be a collection of open sets of $X_{i}$ such that $\{P(j, \alpha): j \in N\}$ forms a neighborhood base of $\alpha \in A_{i}^{\prime \prime}$, $P(j, \alpha) \supset \mathrm{Cl} P(j+1, \alpha)$ for each $j \in N$ and each $\alpha \in A_{i}^{\prime \prime}$, and $\left\{\mathrm{Cl} P(1, \alpha): \alpha \in A_{i}^{\prime \prime}\right\}$ forms a collection of subsets of $X_{i}-A_{i}^{\prime}$ which is discrete in $X_{i}$. Let $\mathscr{T}_{i j}$ be an anticover of $A_{i}^{\prime}$ such that $\mathscr{T}_{i j}<\mathscr{S}_{i} \cup\left\{P(j, \alpha): \alpha \in A_{i}^{\prime \prime}\right\}, \mathscr{T}_{i j}$ is locally finite in $X_{i}-A_{i}^{\prime}$, and mesh $\mathscr{T}_{i j}<1 / j$. Let $L_{i j}$ be the nerve of $\mathscr{T}_{i j}$ and $g_{i j}: X_{i}-A_{i}^{\prime} \rightarrow L_{i j}$ be a Kuratowski map. Let $X_{i j}$ be the disjoint sum of $L_{i j}$ and $A_{i j}^{\prime}$. Define $h_{i j}: X_{i} \rightarrow X_{i j}$ by:

$$
h_{i j} \mid\left(X-A_{i}^{\prime}\right)=g_{i j}, \quad h_{i j}(\alpha)=\alpha, \quad \alpha \in A_{i}^{\prime} .
$$

Give $X_{i j}$ the topology as follows. An open set of $L_{i j}$ is open in $X_{i j}$ and a set $V$ of $X_{i j}$ meeting $A_{i}^{\prime}$ is open if $h_{i j}^{-1}(V)$ is open in $X_{i}$ and if $V-A_{i}^{\prime}$ is the sum of some subcollection of the star-cover $\mathscr{S}_{i j}$ of $L_{i j}$.

Set $P=\bigcup\left\{\mathrm{Cl} P(1, \alpha): \alpha \in A_{i}^{\prime \prime}\right\}$. Let $\alpha$ be an arbitrary element of $A_{i}^{\prime}$ and $\left\{Q_{k}: k \in N\right\}$ be an arbitrary collection of open neighborhoods of $\alpha$ in $X_{i j}$ such that $Q_{k} \supset \bar{Q}_{k+1}(k \in N), \bar{Q}_{1} \cap A_{i}^{\prime}=\{\alpha\}$, and $\bar{Q}_{1} \cap P=\varnothing$. Since $X_{i}$ is not first-countable at $\alpha$ and $\mathscr{S}_{i}$ is approaching to $A_{i}$, there exists an open neighborhood $U$ of $\alpha$ such that $\mathrm{Cl} \mathscr{S}_{i}(U) \cap A_{i}=\{\alpha\}, \mathscr{S}_{i}(U) \cap P=\varnothing$, and $h_{i j}^{-1}\left(Q_{k}\right)-\mathscr{S}_{i}(U) \neq \varnothing$ for any $k \in N$. Set

$$
U^{\prime}=\left(\bigcup\left\{T \in \mathscr{T}_{i j}: T \subset \mathscr{S}_{i}(U)\right\}\right) \cup\{\alpha\} .
$$

Then there exists a set $Q$ of $X_{i j}$ such that $h_{i j}^{-1}(Q)=U^{\prime}$ and $Q-A_{i}^{\prime}$ is the sum of some subcollection of $\mathscr{S}_{i j}$. Since

$$
\mathscr{T}_{i j}\left|\left(X_{i}-P\right)<\mathscr{S}_{i}\right|\left(X_{i}-P\right), \quad U \subset U^{\prime} \subset \mathscr{S}_{i}(U)
$$

and hence $U^{\prime}$ is an open neighborhood of $\alpha$ in $X_{i}$. Thus $Q$ is an open neighborhood of $\alpha$ in $X_{i j}$ with

$$
h_{i j}^{-1}\left(Q_{k}\right)-h_{i j}^{-1}(Q)=h_{i j}^{-1}\left(Q_{k}\right)-U^{\prime} \supset h_{i j}^{-1}\left(Q_{k}\right)-\mathscr{S}_{i}(U) \neq \varnothing .
$$

Therefore $Q_{k}-Q \neq \varnothing$ for any $k \in N$ and $\left\{Q_{k}: k \in N\right\}$ cannot be a neighborhood base of $\alpha$ in $X_{i j}$. Since the first-countability does not hold at any $\alpha \in A_{i}^{\prime}$ in $X_{i j}$, the nonmetric part of $X_{i j}$ is exactly $A_{i}^{\prime}$.

Using the fact that $\mathscr{S}_{i}$ is approaching to $A_{i}$ in $X_{i}$ and that

$$
\mathscr{T}_{i j}\left|\left(X_{i}-P\right)<\mathscr{S}_{i}\right|\left(X_{i}-P\right) \text {, }
$$

it is easy to see that $\mathscr{S}_{i j}$ is approaching to $A_{i}^{\prime}$ in $X_{i j}$. Evidently $X_{i j}$ is paracompact and perfectly normal. Thus $X_{i j}$ is almost polyhedral. 
Define $h_{i}: X_{i} \rightarrow Y_{i}=\prod_{j=1}^{\infty} X_{i j}$ by: $h_{i}(p)=\left(h_{i j}(p): j \in N\right)$. Then by the fact that $\lim _{j \rightarrow \infty}$ mesh $\mathscr{T}_{i j}=0, h_{i}$ is, as can easily be seen, an embedding.

$j \rightarrow \infty$

To complete the proof of the theorem define $\psi_{i}: X \rightarrow Y_{i}$ by: $\psi_{i}=h_{l} g_{i}$, and $\psi: X \rightarrow \prod_{i=1}^{\infty} Y_{i}$ by: $\psi=\left(\psi_{i}\right)$. Then $\psi$ is an embedding into the countable product of almost polyhedral spaces. The proof of the theorem is finished.

The author introduced in [3] the notion of $\mu$-spaces. A space is said to be a $\sigma$-metric space if it is the countable sum of closed metric subsets. A space is said to be a $\mu$-space if it is embedded in the countable product of paracompact $\sigma$-metric spaces. Since each almost metric space is $\sigma$-metric, we get at once the following.

COROLLARY 3.5. The class of free $L$-spaces is a subclass of $\mu$-spaces.

Thus each Lašnev space is a $\mu$-space, which is a new information about Lašnev spaces.

When $\operatorname{dim} X \leqslant 0$, we can assume without loss of generality that order $\mathscr{U}_{i} \leqslant 1$ and order $\mathscr{T}_{i j} \leqslant 1$ in the proof of Theorem 3.4. Then $X_{i j}$ is an almost discrete space. Moreover if $X$ is separable, the cardinalities of $\mathscr{U}_{i}, \mathscr{T}_{i j}$ and $A_{i}$ are countable. Thus we get the following.

Lemma 3.6. Let $X$ be a free $L$-space with $\operatorname{dim} X \leqslant 0$. Then $X$ is embedded in the countable product of almost discrete spaces. Moreover if $X$ is separable, then $X$ is embedded in the countable product of almost discrete countable spaces.

LEMMA 3.7 (Nagami [5], Lemma 3). Let $X$ be the countable product of paracompact $\Sigma$-spaces $X_{i}, i \in N$, with $\operatorname{dim} X_{i} \leqslant 0$. Then $\operatorname{dim} X \leqslant 0$.

The following is a direct consequence of Theorem 3.5, Lemmas 3.6 and 3.7.

THEOREM 3.8. A space $X$ is a (separable) free $L$-space with $\operatorname{dim} X \leqslant 0$ if and only if $X$ is embedded in the countable product of almost discrete (countable) spaces.

\section{References}

[1] I. M. Leibo, On the equality of dimensions for closed images of metric spaces, Dokl. Akad. Nauk SSSR 216 (1974), pp. 498-501; Siviet Math. Dokl. 15 (1974), pp. 835-839.

[2] K. Nagami, Dimension Theory, New York 1970.

[3] - Normality of products, Actes Conırès intern. Math. 2 (1970), pp. 33-37.

[4] - Dimension for $\sigma$-metric spaces, J. Math. Soc. Japan 23 (1971), pp, 123-129.

[5] - Perfect class of spaces, Proc. Japan Acad. 48 (1972), pp. 21-24.

[6] - The equality of dimensions, Fund. Math. 106 (1980), pp. 239-246. 